\title{
Zur Poetik der Pflanzennamen in der Botanik: Carl von Linné
}

\author{
Isabel Kranz \\ Wien \\ isabel.kranz@univie.ac.at
}

„Personne, autant que lui, n’a célébré ou même reconnu le pouvoir du Nom, cœur de la botanique [...]."1

The aim of the following paper is to highlight the poetics of plant names in non-literary texts, namely in the writings of Carl Linnaeus. The first part of the article presents an overview of research on the question of whether plant names have (semantic) meaning. It details the systematics of botanical and vernacular nomenclature and interrogates the relationship between plant names and proper names. The following two parts are devoted to the founder of modern botany, Carl Linnaeus, whose lasting importance lies in systematizing existing plant names as well as establishing a code of rules for future nomenclature. By analyzing a scene in which a plant is (re)named as well as some of the naming rules in his Philosophia botanica (1751), the paper shows that the Swedish nomenclator botanicus already took the poetic dimensions of plant names into account.

\section{Bedeutung als Problem und Herausforderung}

Was für Namen! Salomonssiegel und Aaronstab, Kaiserkrone und Königskerze, Christusdorn und Himmelsschlüssel, die englischen Leopards Bane und Foxglove! In welcher verschollenen und tiefsinnigen Geschichte muss Salomon gesiegelt, der Fuchs Handschuh getragen haben, um das Geheimnis einer Waldblume zu erklären, ist an und in der schönen, so

1 François Dagognet, Le catalogue de la vie: Etude méthodique sur la taxinomie. Paris: PUF, 1970, S. 39 . 
edel sich wiegenden Sternblume des Bergwaldes, Doronicum, der Pardel erstickt (Pardalianches), hat der Fang des Hundes sich in der stillen alpenveilchenartigen Blume wilder Schatten, Erythronium, einverleibt oder einverseelt? ${ }^{2}$

Mit Begeisterung feiert Rudolf Borchardt die Namen der Blumen. ${ }^{3}$

Da sie Erzählungen in knappster Weise verdichten können, scheinen Pflanzennamen für die Literatur besonders ergiebig zu sein. Dank zahlreicher Synonyme lokaler Varianten, sprechender, gelegentlich auch obskurer Bezeichnungen gibt es eine schier unfassbare Vielfalt an Pflanzennamen, wie Borchardt anhand seines kurzen Katalogs demonstriert. Sie überschreiten zudem den Rahmen einer einzigen Sprache: Neben einer Reihe deutscher Blumennamen und zwei (oder drei?) lateinisch-griechischen Beispielen ${ }^{4}$ nennt Borchardt die englischsprachige Bezeichnung für die Fingerhüte (Foxglove) und erwähnt mit Leopards Bane einen umgangssprachlichen Namen, der sich auf unterschiedliche Pflanzengattungen (u. a. Eisenhut und Arnika) beziehen kann. Was in einer Sprache - oder in einem Dialekt - unter einen Begriff fällt, muss also in der anderen nicht zwangsläufig als einheitliche Gruppe bekannt sein. Die Namen der Pflanzen lassen sich daher nicht ohne größeren Aufwand übersetzen, sie bilden vielmehr ein reiches Netz von Verweisen, denen es sich nachzugehen lohnt.

Am Ausgangspunkt der folgenden Betrachtungen steht die zunächst banal erscheinende Beobachtung des grundverschiedenen Zugangs zu Pflanzenamen, ja zur Benennungspraxis überhaupt, den Literatur einerseits und Botanik andererseits haben. Während jene die Namen der Pflanzen bevorzugt

2 Rudolf Borchardt, Der leidenschaftliche Gärtner. Berlin: Matthes \& Seitz, 2016 [1951], S. 19. Der vorliegende Text ist für den 15. Workshop der Allgemeinen und Vergleichenden Literaturwissenschaft zum Thema „Poetik der Namen“ an der Ludwig-Maximilians-Universität München im Februar 2016 geschrieben worden. Frühere Versionen wurden in Erfurt, Wien und Berlin präsentiert. Ich danke den Kolleginnen und Kollegen, die mir geholfen haben, meine Argumente zu präzisieren, sowie insbesondere dem LMUMentoring Programm und Marie Arendt für ihre Unterstützung bei der bibliographischen Recherche.

3 So nennt Borchardt zuvor u. a. die Ovidschen Beispiele von Adonis, Hyazinth und Narkissos und zieht dann eine Linie in der deutschsprachigen Literatur von Goethe und Novalis über Mörike bis hin zu Jean Pauls „Blumengesichte[n]“, „die von wirklicher Pflanzengestalt fast nur noch Namen entlehnen“. Borchardt, Der leidenschaftliche Gärtner (wie Anm. 2), S. 20.

4 Auch die vermeintliche Einsprachigkeit der hier aufgezählten Namen gerät rasch ins Wanken, betrachtet man beispielsweise die Bezeichnung Doronicum: Grammatikalisch den Regeln des Lateinischen folgend, stammt der Begriff ursprünglich wohl aus dem Altpersischen; vgl. Lemma „Dorónicum“, in Helmut Genaust, Etymologisches Wörterbuch der botanischen Pflanzennamen. Hamburg: Nikol, ${ }^{3} 2002$ (Lizenzausgabe der Springer Basel AG), S. $214 \mathrm{f}$. 
zum Sprechen bringt ${ }^{5}$ und aus der besagten Polysemie schöpft, sieht diese in Pflanzennamen eine dauerhaft zu bewältigende Herausforderung im Zeichen einer eindeutigen und transparenten Nomenklatur. Wenn es daher in einem Nachschlagewerk zur Onomastik trocken heißt, „[d]er PflN [i. e. Pflanzenname, I. K.] ist [...] von Anfang an mit dem Bedeutungsproblem belastet", ${ }^{6}$ so liest sich dies geradezu als Einladung an die Adresse der Kultur- und Literaturwissenschaft, sich diesem komplexen und bisher unterschätzten Arbeitsgebiet eingehender zu widmen.

Die folgenden Ausführungen verstehen sich daher als methodische Vorüberlegungen zu einer literarischen Botanik, die zu Anschlusslektüren und weiteren begrifflichen Präzisierungen einladen möchte. ${ }^{7}$ Ziel ist es, poetologische Verfahren der Benennung von Pflanzen in nicht-literarischen Wissenstraditionen aufzuzeigen. ${ }^{8}$ Anhand eines aktuellen Forschungsüberblicks geht es hierbei in einem ersten Abschnitt zunächst um die Frage nach der Bedeutsamkeit von Pflanzennamen sowie deren Systematik und Beziehung zu Personennamen. Die beiden folgenden Kapitel sind dem Gründungsvater der modernen Botanik Carl von Linné gewidmet, dessen herausragende Leistung die Normierung vorhandener Pflanzenbezeichnungen sowie die Etablierung eines Regelwerks zur künftigen Nomenklatur war. In der Lektüre einer Benennungsszene sowie der Richtlinien, die Linné zur systematischen Namensvergabe vorgeschlagen hat, wird sich zeigen, dass die poetischen Dimensionen der Pflanzennamen bereits vom nomenclator botanicus mitgedacht wurden.

5 Vgl. hierzu Isabel Kranz, Sprechende Blumen. Ein ABC der Pflanzensprache. Berlin: Matthes \& Seitz, ${ }^{2} 2016$.

6 Peter Seidensticker, „253. Nomenklaturen: Pflanzennamen“, in: Ernst Eichler u. a. (Hg.), Namenforschung. Ein internationales Handbuch zur Onomastik. Bd. 2, Berlin / New York: de Gruyter, 1996, S. 1622-1626, hier S. 1622; vgl. auch ders., die seltzamen namen all: Studien zur Überlieferung der Pflanzennamen. Stuttgart: Franz Steiner, 1997 (Grundzüge einer historischen Syntax der Pflanzennamen) sowie ders., Pflanzennamen. Überlieferung - Forschungsprobleme Studien. Stuttgart: Franz Steiner, 1999 (weitere Literatur dort). Eine weniger gut lesbare Studie mit einem ausführlichen Beispielteil bietet Friedhelm Sauerhoff, Pflanzennamen im Vergleich. Studien zur Benennungstheorie und Etymologie. Stuttgart: Franz Steiner, 2001.

7 Dies ist eine der zentralen Aufgaben des Forschungsprojekts „Literarische Botanik: Pflanzen als Wissensfiguren 1700 bis 2000", das die Autorin des vorliegenden Aufsatzes seit Herbst 2016 an der Universität Wien, finanziert durch die Fritz Thyssen Stiftung für Wissenschaftsförderung, verfolgt.

8 Ganz im Sinne einer „Poetologie des Wissens“; vgl. Joseph Vogl, „Für eine Poetologie des Wissens“, in: Karl Richter u. a. (Hg.), Die Literatur und die Wissenschaften. Festschrift zum 75. Geburtstag von Walter Müller-Seidel. Stuttgart: M und P Verlag für Wissenschaft und Forschung, 1997, S. 107-127. 


\section{Haben Pflanzennamen Bedeutung und wenn ja, wie viel?}

Wenn eingangs die Rede von Pflanzen- bzw. Blumennamen war, so bedarf diese Bezeichnung einer Klarstellung. Nimmt man gängige Handbücher zur Namenskunde zur Hand, so fällt auf, dass Pflanzennamen oftmals keinen eigenen Eintrag erhalten. Im Oxford Handbook of Names and Naming taucht nicht einmal Carl von Linné im Register auf, und in der Sektion „Other Types of Names“ findet sich zwar neben Einträgen zu Schiffs- und Flugzeugnamen ein Kapitel zu Tiernamen - Pflanzennamen bleiben jedoch ein Desiderat (wobei die Sammelüberschrift schon darauf hinweist, dass im Bereich der Onomastik durchaus noch Systematisierungsarbeit zu leisten ist).

Diese Ausgrenzung der Pflanzennamen beruht auf einer breit akzeptierten Unterscheidung zwischen Eigennamen (nomina propria) als Bezeichnungen für Individuen und Appellativen (nomina appellativa) für Gruppen. In dieser Logik ist es verständlich, dass man von Tiernamen sprechen kann, denn einzelne (wohl eher Haus- als Nutz-)Tiere erhalten durchaus eigene Namen wie der Hund Rollo, ${ }^{9}$ die Katze Minka oder Hansi, der Wellensittich. Individuellen Pflanzen wird jedoch nur selten das Privileg eines eigenen Namens zuteil, ${ }^{10}$ denn gemeinhin "[dient] der PflN, [...] nicht der Identifikation von Individuen, sondern der Klassifizierung. ${ }^{\text {"11 }}$ Dass sich die Unterscheidung zwischen Eigenund Pflanzennamen nicht mit dem einfachen Gegensatz von Identifikation und Klassifikation deckt, wird im Folgenden zu sehen sein.

Doch was ist zunächst genau mit dem Terminus ,Pflanzenname‘ gemeint? Unter diesen Oberbegriff (im Übrigen eine metasprachliche Bezeichnung, die zum ersten Mal im Titel eines Wörterbuchs belegt ist ${ }^{12}$ ) fallen drei verschiedene Gruppen: erstens die vernakularen Pflanzennamen, z. B. die bei Borchardt erwähnten ,Himmelsschlüssel', auch ,Schlüsselblumen' genannt. Diese tragen zweitens einen sogenannten Standardnamen, also jene Bezeichnung, die als Eindeutschung (oder Anglisierung etc.) des botanischen Taxons in deutschen Florenwerken zu finden ist (in diesem Falle wären es ,Primeln'). Primula wiederum ist - drittens - die botanische Bezeichnung für die Gattung der Schlüsselblumen, die dann je nach Art mit einem Epithethon, einem Beiwort

\footnotetext{
$9 \quad$ So heißt der Neufundländer in Theodor Fontanes Roman Effi Briest (1894/1895).

10 Obwohl dies in Literatur und Film durchaus vorkommt: So nennt beispielsweise der Blumenhändler Seymour im Spielfilm Little Shop of Horrors seine fleischfressende Pflanze Audrey II oder auch liebevoll Twoey, sozusagen als zweites, weibliches' Wesen neben der von ihm geliebten menschlichen Audrey. Vgl. Little Shop of Horrors, USA, 1986, Regie: Frank Oz.

11 Seidensticker, Nomenklaturen (wie Anm. 6), S. 1622.

12 Vgl. Seidensticker, Pflanzennamen (wie Anm. 6), S. 16.
} 
oder,Nachnamen', versehen wird, wie z. B. Primula halleri, was als binominaler (oder binärer) Name ein botanisches Taxon bildet (der Standardname dieser Blume wiederum lautet ,Hallerische Primel $\left.{ }^{\prime}\right) .{ }^{13}$

Bis heute existieren vernakulare Pflanzennamen neben den botanischen Taxa und Standardnamen fort. Die Beziehungen zwischen diesen Systemen sind komplex, denn Bezeichnungen können nicht einfach von einem Code in den anderen übersetzt werden. Erstens ist das botanische System weitaus kleinteiliger organisiert als die Namensgebungspraktiken der Amateurwissenschaftler: es dient schließlich dazu, jeder existierenden Pflanzenvarietät einen eindeutigen und stabilen Namen zu verleihen. Aus diesem Grund gibt es weitaus mehr Taxa als Vernakularnamen, und diese "[sind] um ein Vielfaches eindeutiger und aufschlußreicher $[. .$.$] als die entsprechenden dt. Pflanzen-$ namen" ${ }^{14}$ Diese hingegen werden nicht an den gleichen Präzisionsstandards gemessen, denn weder muss jeder vernakulare Name eine einzelne botanische Gattung oder Art bezeichnen wie in der Botanik, wo Homonyme ein Problem darstellen, noch ist das Vorhandensein von mehreren Namen für ein und dieselbe Pflanze, also das Vorhandensein von Synonymen, problematisch. ${ }^{15}$

Im Deutschen tragen die verschiedenen Dialekte und Varietäten überdies dazu bei, dass Pflanzen oftmals verwirrend viele Namen tragen. Was z. B. im Schwäbischen ,Bettsoicher' (Bettnässer) heißt, wird in Franken ,Kuhblume' genanntund bezieht sich in beiden Fällen auf den Löwenzahn, botanisch Taraxacum. Die Problemlage verdichtet sich, wenn man neben den regionalen Varianten nach Entsprechungen von Pflanzennamen in unterschiedlichen

13 Nicht nur semantisch, auch morphologisch betrachtet lehnen sich die Standardnamen an die botanischen Taxa an: „Die Mehrzahl aller Pflanzennamen ist mehrgliedrig, und Linné hat daran mit seiner binären Nomenklatur angeknüpft. Es sind attributive Fügungen, bei denen die modifizierenden Angaben im Epitheton enthalten sind. Als geschlossene semantische Einheiten sind diese Namen im Deutschen auch oft zu Komposita zusammengeschlossen: Lenticula aquatica wird Wasserlinse, Papaver somniferum wird Schlafmohn." Seidensticker, die seltzamen namen all (wie Anm. 6), S. 40.

14 Genaust, Etymologisches Wörterbuch (wie Anm. 4), S. 1. Und die Liste der Taxa verlängert sich stetig, wie Michael Ohl feststellt: „Das Besondere aber [...] ist die Tatsache, dass der größte Teil der in der Natur vorkommenden Gegenstände der biologischen Forschungspraxis noch nicht bekannt ist. Jedes Jahr werden an die 20000 Tier- und Pflanzenarten entdeckt, was wiederum bedeutet, dass der Katalog des Lebens, das Wörterbuch der Natur, jährlich um dieselbe Anzahl von Wörtern erweitert wird, von denen der größte Teil tatsächlich sprachliche Neuschöpfungen sind.“ Michael Ohl, Die Kunst der Benennung. Berlin: Matthes \& Seitz, 2015, S. 89.

15 Um Homonyme und Synonyme zu vermeiden, zirkulieren in Botanik und Zoologie im 17. Jahrhundert Namenslisten, die zu konsultieren Nomenklatoren aufgerufen waren, wollten sie eine neue Art benennen; vgl. Lisbet Koerner, Linnaeus: Nature and Nation. Cambridge, MA / London: Harvard UP, 1999, S. 55 . 
Nationalsprachen zu suchen beginnt. Denn was in einer Sprache unter einen Begriff fällt, bildet in der anderen nicht zwangsläufig eine Einheit. So nennt man im Englischen ,marigold', was im Deutschen sowohl Ringel- als auch Studentenblume sein kann und somit zwei unterschiedlichen botanischen Gattungen (Calendula bzw. Tagetes) angehört. Die drei Namenssysteme, das botanische Latein, die Standardnamen und die jeweiligen Vernakularnamen, sind demnach nicht ohne größeren botanischen und philologischen Aufwand ineinander übertragbar. Umstritten ist zudem, was sie genau unterscheidet.

In zwei ausführlichen Studien zu den botanischen Standardnamen (die vom Autor auch als „Büchernamen“ bezeichnet werden, weil sie Eindeutschungen der Taxa in deutschen Schriftwerken sind) differenziert der österreichische Botaniker Manfred Adalbert Fischer die drei Kategorien in Bezug auf ihre Namenhaftigkeit: Sowohl Standardnamen als auch botanische Taxa gelten für ihn nicht als Eigennamen, sondern als Appellativa zur Bezeichnung von Gruppen, als taxonomische Fachausdrücke, die jenseits ihrer bezeichnenden Funktion nicht semantisch verstanden werden sollten. Denn Fischer ist strikt dagegen, Bedeutung in Pflanzennamen hineinzulesen:

[...] nicht nur die Klientel der deutschen Büchernamen [d. i. Standardnamen, I. K.] sucht [...] krampfhaft nach einem Zusammenhang zwischen Name und Benanntem („nomen est omen“), auch professionelle, florenschreibende Botaniker verfallenen [sic] diesem "psychologischen Zwang“ bei der Beschreibung der Taxa: Nicht selten tauchen bei den Merkmalsangaben nämlich Eigenschaften auf, die bloß durch den Namen des Taxons vorgegeben erscheinen, obwohl es weder eine taxonomische noch logische Notwendigkeit gibt, sie anzuführen, ja, in manchen Fällen sind diese Angaben sogar nichts als irreführend oder gar unrichtig $\left[\ldots . .{ }^{16}\right.$

Fischers rigide Unterteilung in semantisch bedeutsame Namen und vermeintlich bedeutungsfreie Bezeichnungen wird jedoch weder in der Botanik noch in der Onomastik durchgängig geteilt (zumal er ja selbst konzediert, dass in der Mehrzahl der Fälle offensichtlich doch ein motivierter Zusammenhang zwischen Pflanze und Name besteht). Denn in Bezug auf die Frage, ob Eigennamen überhaupt Bedeutung haben können, reichen die Antworten in der Forschung „von strikter Ablehnung über einen Kompromiß (teils - teils, mehr - oder weniger je nach Namenart, ferner: reduzierte Bedeutung) bis zur

16 Manfred Adalbert Fischer, „Wozu deutsche Pflanzennamen?", in: Neilreichia 1 (2001), S. 181-232, hier S. 204-205. 
vollen Zustimmung (Maximum an Bedeutung) “, ${ }^{17}$ wie Gerhard Koß in seinem Beitrag zum Handbuch der Onomastik zusammenfasst.

Da sie von Fischer explizit ausgenommen und auch von Peter Seidensticker gesondert behandelt werden, liegt es nahe zu vermuten, dass sich die Bedeutsamkeit von Pflanzennamen auf Vernakularnamen beschränkt. Schließlich sind sie im Unterschied zu botanischen Taxa sowie den auf ihnen beruhenden Standardnamen nicht von der Gemeinschaft der Botanikerinnen und Botaniker geprägt und in einem regulierten Verfahren akzeptiert worden, sondern in einer langen Tradition aufgekommen. Morphologische Kennzeichen, bekannte medizinische Wirkungen, bevorzugte Lebensräume und mythische Erzählungen sind seit jeher Bezugspunkte für die populäre botanische Namensvergabe. Sie beinhalten ein reiches Wissen über Formen, Funktionen und Eigenarten der Pflanzen, das anhand der Namen rekonstruiert werden kann. Daher bilden die Vernakularnamen denjenigen Teilbereich, in dem semantische Forschungen auch in den Augen streng argumentierender Onomastiker durchaus legitim, ja sogar gewünscht sind. Ausgliedern aus der Diskussion um die Bedeutsamkeit von Pflanzennamen wollen Seidensticker, Fischer et al. hingegen die botanischen Taxa, die sie als nomina appellativa verstehen, bei denen lediglich das Denotat und nicht die Semantik relevant sei. ${ }^{18}$ Dieser Auffassung widerspricht wiederum z. B. Gerhard Bauer, der Namenskategorien im Übergangsbereich von Eigen- zu Gattungsnamen untersucht und insbesondere die botanischen Taxa zu diesen Schwellenphänomenen zählt. ${ }^{19}$

Die offensichtlich ähnliche Struktur von Taxa und Personennamen - beide sind (meistens) binäre Nomen, aufgeteilt in Vor- und Nachnamen - führt hier zu einem weiteren Übergangsbereich. Innerhalb des komplexen Regelwerks der botanischen Nomenklatur kommt nämlich den Personennamen ein gesonderter Status zu. In Botanik und Zoologie ist es Usus, diejenigen, die als Erste eine bestimmte Pflanze oder ein Tier beschrieben haben, nicht aber als deren Namensgeber festgeschrieben wurden, retrospektiv durch einen gültigen Gattungsnamen oder ein Epitheton zu ehren. So hat beispielsweise

17 Gerhard Koß, „67. Die Bedeutung der Eigennamen: Wortbedeutung / Namenbedeutung“, in: Ernst Eichler u. a. (Hg.), Namenforschung. Ein internationales Handbuch zur Onomastik. Bd. 1, Berlin / New York: de Gruyter, 1995, S. 458-463, hier S. 458.

18 Erstaunlicherweise jedoch existiert ein Etymologisches Wörterbuch der botanischen Pflanzennamen (sprich: Taxa), aber bis dato keines der vernakularen Pflanzennamen; vgl. Genaust, Etymologisches Wörterbuch (wie Anm. 4), S. 16.

19 Vgl. Gerhard Bauer, „252. Übergangsformen zwischen Eigennamen und Gattungsnamen“, in: Ernst Eichler u. a. (Hg.), Namenforschung. Ein internationales Handbuch zur Onomastik. Bd. 2, Berlin / New York: de Gruyter, 1996, S. 1616-1621. 
der Botaniker Johann Friedrich Gmelin 1775 eine Schlüsselblumenart dem Schweizer Universalgelehrten Albrecht von Haller zu Ehren Primula halleri benannt. ${ }^{20}$ Neben dieser Möglichkeit der Ehrung gibt es die Konvention, den Namen des Erstbeschreibers in Form eines Autorenkürzels an einen neu etablierten Pflanzennamen anzuhängen. ${ }^{21}$ So lautet das korrekte Taxon der Rosmarinheide Andromeda polifolia, L., wobei das L. für Linné steht. ${ }^{22}$

(Reale oder fiktive) Eigennamen können demnach an drei verschiedenen Positionen des botanischen Taxons genutzt werden: an erster Stelle als Gattungsnamen von Pflanzen (latinisiert, d. h. dekliniert, wie z. B. die Gattung Camissionia zu Ehren von Adalbert von Chamisso, eine Gattung der Nachtkerzengewächse), an zweiter als Epitheton der Art (wie die Arnica chamissoni) oder als Anhängsel an den Pflanzennamen in Form eines Kürzels (Cham. für Chamisso).

Menschliche Eigennamen, die neu entdeckten Pflanzen- und Tierarten vorgängig sind, werden so als Sprachmaterial zur Bildung neuer sogenannter eponymischer Namen genutzt. ${ }^{23}$

Die Verfahren, nach denen Pflanzennamen gebildet werden, zu vereinheitlichen und allgemein gültige Regeln der botanischen Nomenklatur aufzustellen war die Lebensleistung des schwedischen Naturforschers Carl von Linné (1707-1778). Er wird daher in erster Linie als Systematisierer wahrgenommen, während die poetische Dimension seiner Ordnungsarbeit bislang noch nicht ausreichend gewürdigt worden ist. ${ }^{24}$ Im Folgenden werden daher erste

20 Vgl. Johann Friedrich Gmelin, Onomatologia botanica completa, oder Vollständiges botanisches Wörterbuch. Bd. 7, Frankfurt / Leipzig: o. A., 1775, S. 406 f.

21 Vgl. Art. 46 des derzeit gültigen International Code of Nomenclature for algae, fungi, and plants (Shenzhen Code), 2017, https://www.iapt-taxon.org/nomen/main.php?page=title, zuletzt gesehen am 06.07.2018. Diese Praxis existierte laut Staffan Müller-Wille bereits (ohne präzise Regeln) im 18. Jahrhundert; E-Mail-Korrespondenz mit der Autorin am 12.07.2018.

22 Allerdings gilt das Namenskürzel streng genommen nicht als Bestandteil des botanischen / zoologischen Taxons, vgl. Ernst Mayr u. a., Methods and Principles of Systematic Zoology. York, PA: Maple Press Company, 1953, S. 233.

23 Ein ausführliches Kompendium zu dieser Namensgebungsform bietet Lotte Burkhardt, Verzeichnis eponymischer Pflanzennamen. Berlin: Botanischer Garten und Botanisches Museum, 2016, erweiterte Ausgabe 2018, https://www.bgbm.org/de/other-publications/ verzeichnis-eponymischer-pflanzennamen-erweiterte-edition, zuletzt gesehen am 21.01.2019.

24 In der schwedischen Tradition hingegen scheint Linnés literarischer Status weitaus differenzierter gesehen zu werden, als dies die deutsch- und englischsprachige Linnérezeption nahelegt, die deutlich von Michel Foucault geprägt wurde. Zu Linnés unterschiedlichen Facetten vgl. Sten Lindroth, „The Two Faces of Linnaeus“, in: Tore Frängsmyr (Hg.), Linnaeus. The Man and His Work. Berkeley, CA u. a.: U of California P, 1983, S. 1-62. 
Ansätze zu einer Relektüre Linnés im Zeichen der Poetik anhand einer expliziten Benennungsszene sowie einiger Regeln zur Nomenklatur präsentiert.

\section{Die Geburt des Nomenclator botanicus im lappländischen Moor}

Am zwölften Tag seiner Lapplandreise im Jahr 1732 verließ Carl Nilsson Linnaeus seine Unterkunft in Umeå zeitig. ${ }^{25}$ In dunstigem Wetter wanderte er in Richtung Norden. Die Wolken hingen so tief, dass der junge Botaniker "auf einen halben Büchsenschuß nichts sehen konnte“ ${ }^{\text {“26 }}$

Umso stärker ist der Eindruck, den die Begegnung mit einer schüchternen Dame im Moor auf den 24-jährigen machte: „Ich bezweifle, daß ein Maler imstande ist, auf das Bild einer Jungfrau solche Anmut zu übertragen und ihren Wangen solche Schönheit als Schmuck zu verleihen." Was Linnaeus so ins Schwärmen brachte, war jedoch keine Frau aus Fleisch und Blut, sondern eine Blume, von ihm zunächst noch weitschweifig Erica palustris pendula, flore petiolo (im Original petiolio) purpureo genannt. Der Anblick ihrer hängenden Glockenblüten in Zartrosa weckte den Dichter im Forscher. Wie sie so dastand auf einem Stückchen Gras inmitten des Moores, umgeben von Wasser, bedroht von "giftenden Drachen und Getier", erinnerte Erica Linnaeus an Andromeda, Cassiopeias Tochter: Besagte Andromeda war so schön, dass ihre Schönheit sogar Neptuns Töchter bei Weitem übertraf. Solchen Wettstreit konnte der Meeresgott nicht zulassen: Er schmiedete Andromeda an einen Felsen im Meer und schickte ein Ungeheuer, um sie zu töten. Doch Perseus, der Medusenbezwinger, kam der scheuen Schönheit zur Rettung.

In der mythischen Vision des Naturforschers Linné wurden Andromeda und Erica, Frau und Blume, eins: „Da steht sie und läßt voller Traurigkeit ihren Kopf hängen, ihre rosenwangigen capitula florum. Die Wangen werden bleicher und bleicher, capitula pallescunt magis magisque". Und Linnaeus sprach das Zauberwort - von nun an soll die Rosmarinheide den Namen ihrer griechischen Seelenverwandten tragen: „hinc Andromeda dixi, foliis acutis! Sie liegt halb am Boden, der Hals ist bloß, hinc carneus."

"Von nun an nenne ich Dich Andromeda“. In diesem performativen Sprechakt steckt Linnés in den 173oer Jahren noch nicht weiter ausgeführtes

25 Die folgenden Absätze sind angelehnt an den Abschnitt „Andromeda“ in Kranz, Sprechende Blumen (wie Anm. 5), S. 24.

26 Carl von Linné, Lappländische Reise und andere Schriften. Aus dem Schwedischen von H. C. Artmann unter Mitwirkung von Helli Clervall, Leipzig: Reclam, 1987, S. 60 f. Alle folgenden Zitate finden sich an dieser Stelle. 
Programm, der Vielfalt der Pflanzen zu eindeutigen Namen zu verhelfen. Der Naturforscher sieht eine neue Pflanzenart und benennt sie zunächst mit dem ihm bekannten Namen Chamaedaphne Buxb. ${ }^{27}$ In einem zweiten Schritt gibt Linné der Pflanze einen beschreibenden lateinischen Namen (Erica palustris ...), um dann die beiden alten durch einen neuen kurzen Namen zu ersetzen. Einher mit der Neubenennung geht eine Veränderung der Gattungszugehörigkeit: Von nun an handelt es sich bei der Rosmarinheide zwar um ein Heidegewächs, also eine der Familie Ericaceae zugehörige Pflanze, aber eben nicht um eine Torfgränke oder einen Zwerglorbeer (so die beiden deutschen Namen der Chamaedaphne) oder eine Art der Gattung Erica, sondern um die neue Gattung Andromeda (genauer gesagt die Art Andromeda polifolia).

Den neuen Gattungsnamen vergibt Linné mit Bezug auf einen griechischen Mythos, der ihm durch die Gleichsetzung der ,zartrosa Blume' mit einer ,scheuen Frau' in den Sinn kommt. Der Botaniker sieht in den äußeren Merkmalen der Pflanze (Farbe, Wuchs, ihrer Position im Raum) Analogien zu einer erzählten Figur und verleiht ihr deshalb deren Namen. Linnés Träumerei ist hier in ungewöhnlich detaillierter Weise auserzählt, sodass der neue Name als geradezu unumgehbarer Schluss der erotischen Phantasie erscheint. ${ }^{28}$

Wie die Szene aus der Lappländischen Reise zeigt, litt Linné offensichtlich nicht an jener anxiety of influence, die Harold Bloom zufolge junge Dichter oftmals verzweifeln lässt, da alles in der Welt bereits mit Namen versehen ist. ${ }^{29}$

27 Linné schreibt somit die Autorschaft der Chamaedaphne dem deutschen Botaniker Johann Christian Buxbaum (1693-1730) zu. Ebenso wie Linnés neuer Name Andromeda verweist der ältere Chamaedaphne auf die antike Mythologie, wahrscheinlich auf Ovids Metamorphosen, sind doch Ovid, Vergil und Horaz diejenigen Dichter, die Linné in seinen Büchern am häufigsten zitiert; vgl. hierzu John L. Heller, „Classical Poetry in the Systema Naturae of Linnaeus", in: Transactions and Proceedings of the American Philological Association 102 (1971), S. 183-216, hier S. 184 f. und Fußnote 3.

28 In seiner detailreichen Analyse dieser Passage deutet Alain Bewell Linnés implizites Verständnis weiblicher und pflanzlicher Sexualität aus; vgl. Alain Bewell, „,On the Banks of the South Sea': Botany and Sexual Controversy in the Late Eighteenth Century“, in: David Philip Miller / Peter Hanns Reill (Hg.): Visions of Empire. Voyages, Botany, and the Representations of Nature. Cambridge u. a.: Cambridge UP, 1996, S. 173-193, hier S. 177. Dem könnte noch hinzugefügt werden, dass Andromeda ,die an den Mann denkt' bedeutet. Die Namensvergabe durch den träumenden Botaniker, der bei der Blume an die Frau aus dem Mythos denkt, folgt demnach einer Art Zirkelschluss. Staffan Müller-Wille liest diese Szene hingegen als eine Übersetzung unterschiedlicher Wissensbereiche (analytisches und metaphorisches, i. e. poetisches Wissen); vgl. Staffan Müller-Wille, „Joining Lapland and the Topinambes in Flourishing Holland: Center and Periphery in Linnaean Botany“, in: Science in Context 16:4 (2003), S. 461-488, hier S. $465 \mathrm{f}$.

29 "There have been too many Adams, and they have named everything." Harold Bloom, Yeats. Oxford u. a.: Oxford UP, 1970, S. 4. 
Vielmehr tritt der Botaniker selbst als neuer Adam auf, der einem Ding, einer Blume, einen neuen Namen geben und so frühere Namensgeber wie Buxbaum einfach überschreiben kann. Neben einer Kindheitserinnerung ${ }^{30}$ ist die Benennung der Andromeda die zentrale Stelle zum Selbstbild des nomenclator botanicus als poeta doctus. ${ }^{31}$ Die Selbstermächtigungsgeste des schwedischen Naturforschers, in adamitischer Manier einer Pflanze einen neuen Namen zu verleihen, findet in Abwesenheit anderer Menschen mitten im Sumpf statt und steht so im klaren Widerspruch zu den Verfahrensweisen, nach denen ein Pflanzenname seine Gültigkeit gewinnt: Denn ein neuer Gattungsname, so wusste Linné, musste von den Fachkollegen akzeptiert werden. ${ }^{32}$ Seine nachträgliche Verschriftlichung der Lappländischen Reise für die Schwedische Akademie und somit auch der Andromeda-Szene diente daher nicht zuletzt der eigenen Legendenbildung. ${ }^{33}$

Was in der Lappländischen Reise als explizite Benennungsszene ausgestellt wird, findet sich beim späteren Linné nicht mehr in Form einer Erzählung. Doch auch die eher nüchternen Thesen des Nomenklators zur korrekten Benennung der Pflanzen beinhalten poetische Verdichtungen, wie im Folgenden zu sehen sein wird.

Carl von Linnés Schriften markieren eine entscheidende Zäsur in der Wissenschaftsgeschichte: Aufgabe der Naturgeschichte war es künftig, den Erscheinungen der natürlichen Welt eine Ordnung, und das heißt vor allen Dingen: einen Namen zu geben. ${ }^{34}$ Namensgebung war zwar stets Bestandteil

30 Vgl. hierzu Koerner, Linnaeus (wie Anm. 15), S. 21, sowie die fiktionalisierte Szene in Magnus Florin, Der Garten (1995). Aus dem Schwedischen von Benedikt Grabinski, Berlin / Hörby: Edition Rugerup, 2013, S. 20.

31 Zu Linnés literarischer Bildung vgl.: Lindroth, The Two Faces (wie Anm. 24) sowie Koerner, Linnaeus (wie Anm. 15), S. 33-35.

32 Bereits im 18. Jahrhundert waren die Botaniker international gut vernetzt; vgl. hierzu u. a. die Arbeiten von Müller-Wille sowie Bettina Dietz, Das System der Natur. Kollaborative Wissenskultur der Botanik im 18. Jahrhundert. Köln u. a.: Böhlau, 2017.

33 Es gilt zu bedenken, dass Linné seinen Reisebericht retrospektiv ausformulierte und dabei auf literarische Vorgänger zurückgriff: „[...] he presented his Lapland travels of 1732 in the light of Ovidian literature, seventeenth-century Gothicism, early modern anthropology, and neo-Hippocratian environmental medicine." Koerner, Linnaeus (wie Anm. 15), S. 57 .

34 Vgl. hierzu als locus classicus: „Die Naturgeschichte ist nichts anderes als die Benennung des Sichtbaren." Michel Foucault, Die Ordnung der Dinge. Eine Archäologie der 
der Naturgeschichte, ${ }^{35}$ doch erst Linné etablierte die systematische Botanik und Zoologie und rückte die sprachliche Ordnung des Pflanzenreichs sowie deren explizite Reflexion in den Mittelpunkt des Interesses.

In seiner Schrift Systema Naturae von 1735 schlug Linné eine Systematik der Flora vor, die auf Anzahl und Anordnung der Reproduktionsorgane der Pflanzen basiert: Als Indikatoren der Zugehörigkeit einzelner Pflanzen zu Klassen, Ordnungen, Gattungen, Arten und Varietäten bilden sie die Basis seiner Taxonomie. ${ }^{36}$ Indem Linné den Blüten, also den Sexualorganen der Pflanzen, die zentrale Stelle in seinem Klassifikationsschema zuwies, trat die Geschlechtlichkeit in das vormals zumeist als asexuell gedachte Reich der Flora ein; eine einschneidende Veränderung, die in der Wissenschaftsgeschichte bereits hinlänglich diskutiert worden ist. ${ }^{37}$

Darüber hinaus veränderte Linnés klassifikatorisches Projekt das Verhältnis von Populär- und Wissenschaftssprache grundlegend. Linné reformierte die botanische (und später die zoologische) Sprache durch die konsequente Nutzung von binären Namen, ${ }^{38}$ die fortan im wissenschaftlichen Austausch gebraucht werden sollten. Diese Neuerung führte zu einer Pluralisierung

Humanwissenschaften. Aus dem Französischen von Ulrich Köppen, Frankfurt a. M.: Suhrkamp, 1971, S. 173 .

35 Zu botanischen Namensgebungsprozessen in der prä-Linnéschen Botanik vgl. die Studie von Anna Pavord, The Naming of Names. The Search for Order in the World of Plants. London: Bloomsbury, 2005. Eine weiterführende Analyse müsste u. a. die Schriften des Schweizer Botanikers Caspar Bauhin (1560-1624) miteinbeziehen, der vor Linné wichtige Arbeiten zur Vergabe von zweiteiligen Pflanzennamen verfasst hat.

36 Der Begriff Taxonomie wird allerdings erst zu Anfang des 19. Jahrhunderts von Augustin-Pyrame de Candolle eingeführt; vgl. Augustin-Pyrame de Candolle, Théorie élémentaire de la botanique. Paris: Déterville, 1813, S. 19. Carl Ludwig Willdenow spricht hingegen noch von „Systemkunde“; vgl. Carl Ludwig Willdenow, Grundriss der Kräuterkunde: $z u$ Vorlesungen entworfen; mit zehn Kupfertafeln und einer Farbentabelle. Berlin: Haude und Spener, ${ }^{3} 1802$ [1792], S. 219.

37 Vgl. die Arbeiten von Ann B. Shteir und den zentralen Aufsatz von Londa Schiebinger, „The Private Life of Plants“, in: dies.: Nature's Body. Gender in the Making of Modern Science. New Brunswick, NJ: Rutgers UP, 2004, S. 11-39. Zu den Auswirkungen der Entdeckung der Geschlechtlichkeit von Pflanzen in literarischen Texten vgl. u. a. die (allerdings gegen jegliche Gendertheorie abgedichtete) Studie von Amy M. King, Bloom. The Botanical Vernacular in the English Novel. Oxford / New York: Oxford UP, 2003, sowie die umfassende Untersuchung zur Romantik von Theresa M. Kelley, Clandestine Marriage: Botany and Romantic Culture. Baltimore, MD: Johns Hopkins UP, 2012.

38 Die von ihm eingeführten Binomina, heute Taxa genannt, bezeichnete Linné selbst allerdings als „nomina trivialia“ (Koerner, Linnaeus [wie Anm. 15], S.16), was zur weiteren Verwirrung beitragen mag, versteht man heutzutage unter „Trivialnamen“ doch gerade nicht die wissenschaftlichen, sondern die alltagssprachlichen Pflanzen- und Tierbezeichnungen. 
der Pflanzennamen, denn Vernakularnamen und botanische Taxa sowie die längeren beschreibenden Namen existierten nebeneinander; ${ }^{39}$ hinzu kamen etwas später die nationalsprachlichen Standardnamen. Eine Pflanze trägt also auch nach Linné weiterhin viele Namen - geregelt war fortan lediglich die entscheidende Frage, nach welchen Regeln sie im Fachdiskurs benannt werden soll, der in standardisiertem botanischen Latein, „einem supranationalen Sprachsystem ", 40 abgehalten wird.

Linnés wichtige Werke zur Namensgebung stammen aus den 1750er Jahren. Die Schrift Species plantarum (1753), in der er erstmals alle ihm bekannten Pflanzen mit binominalen Namen (also einer Bezeichnung für die Gattung und einem begleitenden Epithet für die Art, wie z. B. Bellis perennis, Gänseblümchen) versah, gilt für die Botanik als terminus post quem, ${ }^{41}$ während für die Tiernamen die zehnte Auflage seines Systema Naturae (1758), also eine spätere Schrift, ausschlaggebend ist. Für eine ausführliche Diskussion zur Namensgebung lohnt es sich jedoch, Linnés frühere Philosophia botanica von $175^{1} \mathrm{zu}$ Rate zu ziehen. Hier fasst Linné einige seiner vorhergehenden Publikationen zusammen, wie z. B. die Bibliotheca botanica (1735), eine kommentierte Bibliographie der wichtigsten botanischen Schriften, oder die Critica botanica (1737), in der er zum ersten Mal die Grundregeln für die korrekte Namensgebung skizzierte. Die Philosophia ist wie ein Almanach - in 12 Kapitel und 365 Einträge - gegliedert. Anders als der Bericht an die Schwedische Akademie, der retrospektiv die Geburt des Botanikers aus dem Geist der (Kolonial-)Reise schildert und somit auch eine Rechtfertigung für die Finanzierung dieser Unternehmung liefern will, sind die Philosophia und andere Schriften didaktisch motivierte Gebrauchs- und Lehrwerke, die vor allen Dingen mnemotechnisch ausgerichtet sind. Dies zeigt sich am aphoristisch verkürzenden Schreibstil sowie einem komplexen internen Verweissystem. ${ }^{42}$ Erweitert um zahlreiche

39 Denn die botanische Nomenklatur war nicht auf die Ersetzung der beschreibenden Namen ausgerichtet, sondern auf deren Ergänzung, vgl. William T. Stearn, „The Background of Linnaeus's Contributions to the Nomenclature and Methods of Systematic Biology“, in: Systematic Zoology 8:1 (1959), S. 4-22.

40 Diese Formulierung stammt von Genaust, Etymologisches Wörterbuch (wie Anm. 4), S. 1. $\mathrm{Zu}$ den Eigenarten des botanischen Latein vgl. William T. Stearn, Botanical Latin. Newton Abbot / Portland, OR: David \& Charles / Timberpress, ${ }^{4} 2008$.

41 Auf dem Internationalen Botanischen Kongress in Wien 1905 wurden alle Pflanzennamen, die vor 1753 vergeben wurden, für wissenschaftlich ungültig erklärt. Vgl. Lorraine Daston, „Type Specimen and Scientific Memory“, in: Critical Inquiry 31:1 (2004), S. 153-182, hier S. 162.

42 Zu Linnés Schreibstil vgl. Staffan Müller-Wille / Karen Reeds, „A translation of Carl Linnaeus's introduction to Genera plantarum (1737)“, in: Stud. Hist. Phil. Biol. \& Biomed. Sci. 38 (2007), S. 563-572, hier S. 564. 
Beispiele aus seinen Vorlesungen, war Linnés Philosophia als Kondensat der Botanik zunächst ganz pragmatisch für seine Studenten gedacht, wird heute jedoch als wichtigstes theoretisches Werk des Schweden angesehen. ${ }^{43}$

Zunächst würdigt Linné im Abschnitt Bibliotheca (I.) seine Vorgänger und präsentiert deren Ordnungssysteme (Systemata) (II.), definiert danach Pflanzen (Plantae) (III.) und stellt ihre Fruchtkörper (Fructificatio) (IV.) und Fortpflanzungsmechanismen (Sexus) (V.) und die darauf basierenden Merkmale (Characteres) (VI.) vor. Beginnend mit dem siebten Abschnitt geht es dann um die Etablierung der Namen. Welche zentrale Rolle sie für den Botaniker spielen, zeigt sich bereits im 7. Aphorismus des gesamten Buchs, in dem es heißt, die „echten Botaniker“ wiesen sich dadurch aus, dass sie alle Pflanzen mit verständlichen Namen zu bezeichnen wissen (vegetabilia omnia nomine intelligibili nominare sciant ${ }^{44}$ ). In den jeweiligen Abschnitten zu den Namen (VII. Nomina) und den Beschreibungen (VIII. Differentiae) wird dann genauer ausgeführt, was es heißen soll, korrekt zu benennen.

Zunächst sind Namen unerlässlich, um das Gebiet der Botanik, das Linné wahlweise als Republica Botanica (§ 221) oder forum Botanicum ( $(226)$ bezeichnet, möglichst effizient zu bearbeiten. Linné versucht hierzu strenge Regeln einzuführen, denn „Privatleute haben absurde Namen vergeben“ (Idiotce imposuere nomina absurda ${ }^{45}$ ). Deshalb sind die Botaniker (und nur diese) zur Namensvergabe aufgerufen. Ihre Aufgabe ist es, zu ordnen und zu benennen: „51. FUNDAMENTUM Botanices (4) duplex est: Dispositio \& Denominatio“46 Die Ordnung des Pflanzenreichs in Klassen, Ordnungen, Familien, Gattungen und Arten basierend auf der entsprechenden Anzahl, den Proportionen und Positionen der männlichen und weiblichen Teile der Pflanzenblüten, ist demnach nur die Hälfte der Arbeit. Die andere ist das Aufstellen einer Nomenklatur, denn ohne Namen ist alles andere umsonst, wie es bei Linné heißt: „Wenn man die Namen der Dinge nicht weiß, so geht das Wissen von ihnen ebenfalls verloren“ (Nomina si nescis, perit \& cognitio rerum $^{47}$ ). Namen sind demnach unerlässlich für die Tradierung von Wissen,

43 Vgl. Koerner, Linnaeus (wie Anm. 15), S. 40.

44 Carl von Linné, Philosophia botanica. Stockholm: Godofr. Kiesewetter, 1751, S. 4. Da trotz des durchschlagenden Erfolgs der Philosophia bis heute keine vollständige deutsche Ausgabe existiert, stammen alle folgenden Übersetzungen von mir, I. K.

45 Linné, Philosophia botanica (wie Anm. 44), S. 158.

46 Linné, Philosophia botanica (wie Anm. 44), S. 97, Herv. i. O.

47 Linné, Philosophia botanica (wie Anm. 44) S. 158. Dieser zentrale Merkspruch ist ein Hexameter, das Metrum dient hier der leichteren Memorierbarkeit. Vermutlich hatte Linné in der Schule gelernt, lateinische Gedichte zu verfassen. Für diesen und weitere wichtige Hinweise (Anm. 62) danke ich Martin Korenjak. 
sprich: die Möglichkeit von (Natur-)Geschichte. Hierzu leistete Linnés Durchsetzung des binominalen Systems der botanischen (und später zoologischen) Namensvergabe einen entscheidenden Beitrag. ${ }^{48}$

In der Philosophia botanica stellt er die Regeln auf, nach denen die beiden Teile eines Taxons gebildet werden. Die Gattungsbezeichnung, der erste Teil des Namens, kann dabei unterschiedlicher Herkunft sein. Ganz Pragmatiker versucht Linné, an möglichst vielen der bereits vorhandenen Gattungen festzuhalten. Allerdings darf der Gattungsname nur aus einem einzelnen Wort bestehen, dieses darf nicht als Fachterminus in anderen Wissensbereichen gebraucht werden (medizinische, anatomische Bezeichnungen etc. werden ausgeschlossen), Substantive werden gegenüber Adjektiven bevorzugt und Diminutive gerade noch toleriert (obwohl sie "nicht sehr gut", non optima, $\left.\operatorname{sind}^{49}\right)$. Zu vermeiden sind Namen, die Heilige oder historische Persönlichkeiten verewigen. Gattungsnamen, die der Dichtung entstammen - vorzugsweise der griechischen und römischen Mythologie wie Andromeda -, dürfen hingegen beibehalten werden, ebenso jene, die einen hervorragenden Botaniker verewigen: Sie müssen Linné zufolge geradezu religiös behandelt werden $\left(\S 238\right.$, sancte servanda sunt $\left.{ }^{50}\right)$, denn sie sind die einzige Ehre, die Botaniker erhalten können.

Die neu zu bildenden Gattungsnamen, so führt Linné im 240. Aphorismus aus, sollten wenn möglich die äußere Gestalt (habitus) oder den wesentlichen Charakter (character essentialis) der Pflanze bezeichnen. Angestrebtes Idealziel ist ein Name, der "das Unterscheidende derselben [Gattung, I. K.] von allen übrigen enthält" ${ }^{\text {, }}$,1 wie Carl Ludwig Willdenow 1802 übersetzt. ${ }^{52}$ Wie bei all seinen Lehrsätze erläutert Linné seine Forderung anhand langer Tabellen

48 Wohlgemerkt in der Durchsetzung, nicht der Erfindung; vgl. Pavord, The Naming of Names (wie Anm. 35), S. 396.

49 Linné, Philosophia botanica (wie Anm. 44), S. 167.

5o Linné, Philosophia botanica (wie Anm. 44), S. 171. Es gilt jedoch bis heute als unfein unter Biologen, Pflanzen oder Tiere mit dem eigenen Personennamen zu versehen: Üblich sind Reverenzgesten gegenüber Vorgängern, Mentoren etc. Linné selbst hat allerdings die Linnaea borealis, das Moosglöckchen, nach sich benennen lassen, und zeigte sich mit ihr auf vielen der von ihm bekannten Porträts; vgl. Koerner, Linnaeus (wie Anm. 15), S. 18.

$5^{1} \quad$ Willdenow, Grundriss der Kräuterkunde (wie Anm. 36), S. 221.

52 Linné, Philosophia botanica (wie Anm. 44), S. 176. Die Suchbewegung nach dem besten aller möglichen Namen ist schon bei Linné als tendenziell unendlich angelegt und wird aus pragmatischen Gründen abgebrochen, sobald ein arbeitsfähiger Name gefunden worden ist: Sed abstinendum ab hac innovatione, quce nunquam cessaret, quin indies aptiora detegerentur in infinitum. § 243, Linné, Philosophia botanica (wie Anm. 44), S. 196. Linné konzediert jedoch, dass dies nur selten gelingt: Character Essentialis raro in plantis obtinetur, licet optimus. 
von Pflanzennamen. ${ }^{53}$ Zur Anschaulichkeit seiner These werden besonders gelungene Namen vom Griechischen ins Lateinische übersetzt, indem beispielsweise Komposita in ihre Wortbestandteile zerlegt werden wie im Fall

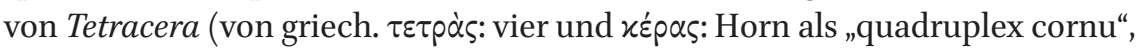
also vierfaches Horn) oder Triopteris („triplex ala“, also Dreiflügel). ${ }^{54}$ Viele dieser griechischen Namen folgen laut Linné dem Prinzip Habitus indicat similitudinem, qua excitatur idea, \& ex idea nomen ${ }^{55}$ („Die Gestalt verweist auf eine Ähnlichkeit, die eine Vorstellung hervorruft, und aus der Vorstellung [entstammt] der Name"). Der Großteil der beispielhaft aufgezählten Namen wird auf Basis eines besonderen Merkmals der Pflanze gebildet, wenn beispielsweise der süße Geschmack der Wurzeln den Namen evoziert (Glycyrrhiza von

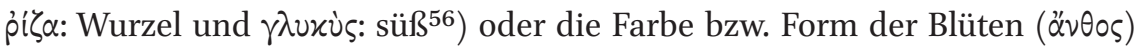
wie bei Melanthium (schwarz) oder Sphaeranthus (rund). Komplexer wird die Namensgebung, wenn Tiere zum Vergleich herangezogen werden (wie im Fall von Myosotis, ${ }^{57}$ von $\mu$ ús: Maus und oîs: Ohr, oder Leontice, ${ }^{58}$ Löwenzahn) oder der menschliche Körper (wie bei Hoemanthus, ${ }^{59}$ blutrote Blume, oder Rhinanthus ${ }^{60}$ nasenförmige Blume) oder andere Bildbereiche (vgl. Helianthus, sonnenförmige Blüte).

Anna Pavords These, post-Linnésche Namen müssten nur noch bezeichnen („designate“) und nicht mehr beschreiben („describe“), ${ }^{61}$ muss also relativiert werden: Denn seit Linné sind zwar die beschreibenden Namen weniger relevant als konzise und kurze Namen; die Gattungsnamen sollten aber im

53 Während sie Linné als mnemotechnische Hilfe für seine Studenten anführt und sie zunächst nur als Ausweis botanischer Bildung gelten mögen, scheinen gerade diese auf den ersten Blick so nüchternen Listen von Pflanzennamen für die Literatur von besonderem ästhetischem Mehrwert zu sein, wie u. a. William Stowe in Bezug auf die amerikanischen Transzendentalisten bemerkt (was im Übrigen für viele Literaten, auch den eingangs zitierten Borchardt, gilt): „[...] the endless lists of such names to be found in the works of eighteenth-century naturalists foster a feeling of airless erudition and an assumption of superiority to nature. For a long time I skipped them myself, and advised students to do the same. Then I read one of William Bartram's lists aloud and was struck by the incantatory quality of the sound it produced, and the sheer pleasure of performing it." William Stowe, "Linnaean Poetics: Emerson, Cooper, Thoreau, and the Names of Plants“, in: Interdisciplinary Studies in Literature and Environment 17:3 (2010), S. 567-583, hier S. 567 .

54 Diese und alle folgenden griechischen Schreibungen wie bei Linné, Philosophia botanica (wie Anm. 44), S. 176.

55 Ebd.

56 Ebd.

57 Linné, Philosophia botanica (wie Anm. 44), S 179.

58 Linné, Philosophia botanica (wie Anm. 44), S 180.

59 Linné, Philosophia botanica (wie Anm. 44), S 177.

6o Ebd.

61 Vgl. Pavord, The Naming of Names (wie Anm. 35), S. 396. 
besten Falle ein herausragendes, wenn nicht gar das differenzierende Merkmal der jeweiligen Pflanze benennen, d. h. mimetisch, geradezu kratylisch funktionieren. In seiner Suche nach den bestmöglichen Namen nutzt Linné die Potentiale der Etymologie, um seinen Studenten gelungene Beispiele aus der griechischen Sprache zu erläutern. Und offensichtlich ist es kein Hinderungsgrund, wenn diese auf Basis von Tropen (pars pro toto, Metaphern) gebildet wurden.

Das Begehren nach besonders treffenden Namen lässt sich an anderen Stellen in der Philosophia ebenfalls aufzeigen. Neben pragmatischen Überlegungen finden sich dezidiert ästhetische Urteile. Sie beziehen sich nicht nur auf die Verschriftlichung, sondern auch auf den Klang der neu zu bildenden Namen. So sollten u. a. allzu lange Namen (wie Coriotragematodendros oder Hypophyllocarpodendron, vermieden werden - das Maß ist anderthalb Fuß $§ 249$, nomina sesquipedalia ${ }^{62}$ ) - da sie schwierig auszusprechen und geradezu übelerregend (nauseabunda) seien, und auch generell sollte die Aussprache von Gattungsnamen erleichtert werden. Hybride Namensgebungen (wie Chrysanthemindum oder Cardamindum) lehnt Linné ab, und Namen, die aus anderen als den antiken Sprachen Latein und Griechisch (§ 229) stammen und sich bereits etabliert haben, werden durchgängig als lateinische Wörter behandelt, das heißt also der lateinischen Grammatik entsprechend dekliniert.

Bei einer Eingemeindung fremder Namen in das botanische Latein will Linné, der selbst keine moderne Fremdsprache außer seiner Muttersprache Schwedisch aktiv beherrschte, es aber erstaunlicherweise nicht belassen. So heißt es im selben Abschnitt weiter unten:

„Wir übernehmen barbarische Namen, als ob sie neu geboren würden, wenn wir die auszuschließenden Wörter neu machen, indem wir sie aus dem Griechischen oder Lateinischen formen"(Quasimodogenita assuminus nomina Barbara, dum vocabula excludenda nova reddimus, formata e lingua grceca, aut latina ${ }^{63}$ ). Die hier angesprochene Adoption (assumere) von Fremdwörtern ist also nicht allein durch deren grammatikalische Angleichung vollendet, vielmehr erschafft Linné sie neu auf der Ebene der Semantik:

Thea (Sinensibus) $\theta \varepsilon \dot{\alpha}$ Dea.

Coffea (Arabibus) $\varkappa \omega \varphi \dot{\varepsilon} \omega$ [sic] obmutesco.

Musa (Arabibus) Musa Anton.

Cassine (Americanis) « $\alpha \sigma \sigma \dot{\omega} \omega$ machino.

62 Eine Referenz an Horaz, der in der Ars poetica über Dichter spottet, die überlange Neologismen erfinden.

63 Linné, Philosophia botanica (wie Anm. 44), S. 163. 
$[\ldots]$

Mammea (Mammel Americ.) a fructu mammoso.

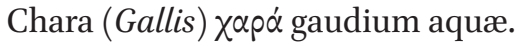

Wie zuvor erläutert, bedient sich Linné auch andernorts der Etymologie. Hier jedoch setzt er dieses Verfahren ein, obwohl es offensichtlich keine philologische Grundlage gibt. Schließlich legt Linné offen, dass die oben genannten Bezeichnungen gerade nicht aus den antiken Sprachen stammen, sondern nomina Barbara sind. Namensgebung wird so zu einem Akt, der den Wörtern einen anderen Ursprung im Griechischen bzw. Lateinischen oder in den Eigennamen bekannter Botaniker wie Antonio Musa (anstelle des arabischen Worts musa) zuweist, also eine fiktive Etymologie aufstellt. Diese Rekodierungen basieren auf der rhetorischen Figur der Paranomasie, auch Annominatio genannt, laut Heinrich Lausberg ein "(pseudo-)etymologisches Spiel mit der Geringfügigkeit der lautlichen Änderung einerseits und der interessanten Bedeutungsspanne, die durch die lautliche Änderung herausgestellt wird, andererseits. "64 Linné buchstabiert Paranomasien aus, indem er hinter die nicht-lateinischen und nicht-griechischen Pflanzennamen jeweils einen Terminus aus diesen Sprachen setzt, der diesen lautlich nahe kommt. ${ }^{65}$ So wird der Gattung Chara, deren Name laut Linné aus dem Französischen stammt, das griechische Wort $\chi \alpha$ pó nachgestellt (zu Deutsch „Freude“) und der Name lateinisch als gaudium aquae, wörtlich „Freude am Wasser“, paraphrasiert, was für eine Alge ein äußerst passender Name ist. Auf ähnliche Weise wird der chinesische Begriff ,Tee' mit dem Wort ,Göttin' (dea) assoziiert, ${ }^{66}$ dem arabischen Namen ,Kaffee ${ }^{`}$ wird seine betäubende (möglicherweise Appetit unterdrückende) Bedeutung eingeschrieben ( $\varkappa \omega \varphi \hat{\varepsilon} \omega$ bzw. obmutesco: ich werde stumm), und der amerikanische Name Mammea für eine von Linné selbst etablierte Gattung erhält einen Hinweis auf die Form der Frucht, nämlich dem weiblichen Busen ähnlich (von lat. mamma). Am Ende der Linnéschen Pseudo-Etymologien stehen also neu geschaffene Pflanzennamen

64 Heinrich Lausberg, Handbuch der literarischen Rhetorik: Eine Grundlegung der Literaturwissenschaft. Stuttgart: Steiner, ${ }^{3} 1990$, S. 322 . Ich danke Fabienne Imlinger für diesen Hinweis.

65 In einer ausführlicheren Studie wäre nachzuzeichnen, welche Transkriptions- und Aufzeichnungsprozesse die hier genannten Namen durchlaufen haben.

66 Zum Vorwurf des linguistischen Imperialismus, der Linné in diesem Zusammenhang häufig gemacht wird, vgl. die Ausführungen über Tee in Alexandra Cook, „Linnaeus and Chinese Plants: A Test of the Linguistic Imperialism Thesis", in: Notes and Records of the Royal Society of London 64 (2010), Nr. 2, S. 121-138, hier S. 124 f. 
(quasimodogenita), denen jenseits ihrer bloßen Zeigefunktion Bedeutungen eingeschrieben worden sind.

An dieser Stelle finden wir deutliche Anzeichen dafür, dass Linnés Ausführungen zur Namensgebung in der Philosophia botanica keine reine Stilistik sind, wie man es aufgrund der Ablehnung von hybriden oder allzu langen Namen aus ästhetischen Gründen vermuten könnte, sondern Ansätze zu einer Poetik aufweisen. Schließlich gibt Linné nicht nur die Regeln zur Bildung neuer Namen vor, sondern er erschafft bereits existierende Namen neu (quasimodogenita von gignere, erschaffen, das lateinische Äqui-

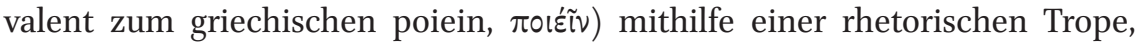
der Paranomasie, die auf Basis einer fiktiven Etymologie zu einer neuen Bedeutungszuschreibung führt. Das Bestreben, eine sprachlich homogene Oberfläche des Lateinischen (mit einer Tiefenstruktur im Griechischen) zu bewahren, geht so einher mit der Produktion von sinnhaften, d. h. semantisch motivierten Namen. Selbst Linné, der oftmals als nüchterner Systematisierer verstanden wird, kann sich also eines kratylischen Begehrens nicht erwehren.

Das hier geschilderte Moment einer produktiven onomaturgischen Freiheit lässt sich gegen den in der Forschung oftmals betonten anti-rhetorischen Gestus Linnés ins Feld führen, der in $§ 292$ formuliert wird, wenn es in Bezug auf die Bildung von Artnamen heißt: „Ausgeschlossen seien Antonomasie [eine Form von Metonymie, I. K.], Tautologie \& rhetorische Floskeln“ (Exulent Antonomasia, Tautologia \& flosculi oratorii $\left.{ }^{67}\right)$. Vier Aphorismen (§ 296) später wird ausgeführt:

Der Artname [nomen specificum, I. K.] darf nicht mit rhetorischen Figuren geschmückt werden; noch weniger soll er falsch sein, sondern er soll getreu wiedergeben, was die Natur diktiert.

Synekdoche eines Teils für das Ganze ist häufig in der Botanik, wo für das Ganze gefordert wird, was nur für einen Teil stimmt; was unserer Meinung nach nirgendwo zugelassen werden sollte.

Synekdoche des Singulars für den Plural ist sehr häufig und gleich fehlerhaft.

Eine Metapher bleibt immer dunkel, zu bevorzugen ist die nackte Einfachheit.

Da die Ironie eine Art von Lüge ist, muss sie ausgeschlossen werden. ${ }^{68}$

67 Linné, Philosophia botanica (wie Anm. 44), S. 229.

68 "Nomen specificum non erit Tropis Rhetoricis figuratum, multo minus erroneum, sed fideliter, quæ natura dictitat, exponat. Synecdoche totius pro parte frequentissima est in 
Rhetorische Tropen in der Namensgebung werden also explizit abgelehnt, wenn die durch sie getroffenen Aussagen der Morphologie der Pflanzen widersprechen oder wenn sie reiner Zierrat sind. Linné nennt hier beispielsweise den Namen Salicaria purpurea (Blutweiderich, mittlerweile Lythrum salicaria), dessen Artepitheton suggeriert, die ganze Pflanze sei purpurfarben, während dies nur für die Blumenkrone zutrifft (Synekdoche des Ganzen für einen Teil), und Hesperis melancholica (Nachtviole), deren Beiname auf ihr, Temperament ${ }^{\star}$ verweist, also metaphorisch funktioniert, während eine präzise Beschreibung sich auf den Duft der Blüten beziehen würde (die nachts stärker als tagsüber riechen). Durchaus eingesetzt werden Tropen hingegen in der Bildung von Gattungsnamen, wenn dies der Eingemeindung existierender Namen in die klassischen Sprachen und dadurch der Etablierung von Bedeutung dient. Rhetorische Stilfiguren sind demnach keine Fremdkörper im nomenklatorischen System, die generell ausgeschlossen werden, sondern vielmehr Teil dieses Regelwerks, deren Einsatz bestimmten Richtlinien folgen muss.

Namen sind also für Linné weitaus mehr als bloße Etiketten, die einer Pflanze lediglich einen bestimmten Platz innerhalb der Taxonomie zuweisen, sie sind auch sprachliche Zeichen. Taxonomie und Nomenklatur erweisen sich hier als verschränkte, aber voneinander deutlich zu unterscheidende Arbeitsbereiche: Geht es in der Taxonomie um die (Ein)Ordnung von Arten, Gattungen etc., so behandelt die Nomenklatur die Frage, wie ein korrekter Name zu bilden ist und wann ein solcher vergeben werden kann bzw. muss. ${ }^{69}$ Jeder Nomenklatur wohnt also schon immer ein Anteil von präskriptiver Poetik inne. Linné geht jedoch weiter, indem er nicht nur Regeln zur Bildung oder Bewahrung richtiger oder ästhetisch angenehmer Namen aufstellt, sondern bereits vorhandenen Namen eine neue Bedeutungsschicht einträgt, sie so retrospektiv zu sprechenden Namen macht.

Botanicis, ubi de toto prædicatur, quod valet de parte tantum; quod nunquam admittendum esse censemus. Synecdoche numeri singularis pro plurali tritissima \& æque erronea est. Metaphora semper obscura est, hinc præstat nuda simplicitas. Ironia cum mendacii species sit, hinc excludenda erit." Linné, Philosophia botanica (wie Anm. 44), S. 231. 
„Il nous faut ressaisir ce moment assez privilégié, où les naturalistes se préoccupent autant des mots que des fleurs."70

Die vorangegangenen kursorischen Ausführungen haben gezeigt, dass sich im Übergangsbereich zwischen Literatur und Botanik ein reiches Forschungsfeld auftut, das bis dato erst in seinen Konturen erkennbar ist. ${ }^{71}$ Als vielversprechender Ausgangspunkt für zukünftige Forschungen erweisen sich die Pflanzennamen, die in ihrer Nähe zu und ihrer Verbindung mit menschlichen (realen oder fiktiven) Eigennamen und dank ihrer ästhetischen Qualitäten einer ausführlichen Würdigung harren. Hierzu lohnt es sich, den Mechanismen der Nomenklatur am Übergang einer vornehmlich medizinisch orientierten Pflanzenkunde zur Botanik als einem ordnungsgebendem sprachbasierten System, dessen wichtigste Operatoren die Namen sind, nachzugehen, und zwar mit literaturwissenschaftlichen Methoden. Schließlich sind Botaniker (in der frühen Neuzeit fast ausschließlich Männer), wie Lorraine Daston pointiert formuliert, Autoren: „Botanists may not create their species, but they author them in a strong sense, belied by the figurative but borne out by the literal sense of a 'nominal author' - here, the author of a name “. ${ }^{72}$ Dementsprechend wohnt ihren Arbeitsprozessen eine Poetik inne, die sich anhand der Pflanzennamen erschließen lässt.

Als ein erstes Fallbeispiel konnte im Rückgriff auf Linné gezeigt werden, dass die Poetizität der Phytonyme kein Effekt ihrer Aufnahme in literarische Texte ist, vielmehr existierte bereits am Beginn der neuzeitlichen Botanik eine Poetik der Pflanzennamen. Linnés Philosophia botanica, die als Einführung in die Grundlagen der Pflanzenkunde angelegt ist und die Regeln seiner Nomenklatur festhält, beinhaltet ästhetisch-stilistische Reflexionen ebenso wie Momente dichterischer Freiheit. So bedient sich der wichtigste Namensgeber der Botanik trotz gegenteiliger Ansagen durchaus poetischer Verfahren, indem er nicht nur Namen systematisch vergibt, die er bereits vorfindet, sondern auch

70 Dagognet, Le catalogue de la vie (wie Anm. 1), S. 19.

71 Für einen ersten Überblick vgl. die Einleitung „Das literarische Leben der Pflanzen: Poetiken des Botanischen“ im gleichnamigen Themenheft, Joela Jacobs / Isabel Kranz (Hg.), Literatur für Leser, 17:2 (2017, ersch. im August 2019). Zu einer systematischeren Aufbereitung des historischen Pflanzenwissens für die Geisteswissenschaften wird das Nachschlagewerk Pflanzen: Ein kulturwissenschaftliches Handbuch dienen, das Isabel Kranz und Joela Jacobs für 2021 im Metzler Verlag vorbereiten. Für aktuelle Veranstaltungen sowie einen Überblick über die Forschung im Bereich der literaturwissenschaftlichen Pflanzenstudien s. das Literary and Cultural Plant Studies Network an der University of Tucson, Arizona unter https://plants.arizona.edu/, zuletzt gesehen am 02.02.2019. 
nach eigens erstellten Regeln Namen (neu) erfindet und vorhandenen Namen Bedeutung einschreibt. ${ }^{73} \mathrm{Im}$ einen wie im anderen Fall sind rhetorische Tropen an der Bildung der Namen beteiligt, auch wenn diese gezielt aus dem nomenklatorischen System verbannt werden sollen. Linnés Versuch, ausschließlich über botanische Blumen zu sprechen unter Ausschluss rhetorischer Blümchen (flosculi oratorii) wird demnach von den Tropen heimgesucht.

Ein besonderer Status kommt in diesen Prozessen der Etymologie zu, und zwar als Methode und als Untersuchungsgegenstand. Als erstere wird sie von der Linguistik bevorzugt zur Analyse von Pflanzennamen eingesetzt. Die Aufschlüsselung der Wortbestandteile führt oftmals zu hilfreichen Erläuterungen bezüglich der Pflanzenverbreitung, ihres medizinischen und kultischen Nutzens und nicht zuletzt zu Informationen über die Botanikgeschichte. Allerdings scheitert die Sprachwissenschaft mit ihrem Urvertrauen in die Etymologie an eben jenen Punkten, an denen Pflanzennamen nicht mehr auf vermeintlich eindeutige Ursprünge zurückzuführen sind und somit zu rätselhaften Zeichen werden.

Besonders anschaulich zeigt dies Helmut Genaust, wenn er in der Einleitung zu seinem (immens hilfreichen) Etymologischen Wörterbuch der botanischen Pflanzennamen folgendes Beispiel anführt, das uns zurück zu Rudolf Borchardts Begeisterung für die Phytonyme bringt:

Denn wie soll man beispielsweise eine so harmlose Zier- und Heilpflanze wie die in Südwest- und Mitteleuropa heimische Kriech-Gemswurz beurteilen, die zufolge des ihr von Linné verliehenen Artnamens Doronicum pardalianches angeblich den Panther würgt? Jeder vernünftige Mensch weiß, daß selbst dann, wenn tatsächlich einmal ein Panther durch die Wuchsgebiete dieser Art streifen sollte, diese nie und nimmer imstande wäre, die Raubkatze in den Würgegriff zu nehmen; und außer der berühmten Boa in Saint-Exupérys Kleinem Prinzen kenne ich auch kein anderes Geschöpf, das sich dieser undankbaren Aufgabe unterzogen hätte. Im wesentlichen ist es wohl die mangelhafte Kenntnis der Benennungsgeschichte, die weniger den Autor selbst als den Interpreten, sei er nun Linguist oder ausgewiesener Botaniker, zu solch verstiegenen Behauptungen zwingt. ${ }^{74}$

73 Zur Notwendigkeit der Neubildung von Wörtern, vor allem von Nomina, im frühneuzeitlichen Latein vgl. Hans Helander, „Why was it Necessary for Neo-Latin Authors to Coin New Words?“, in: Steiner-Weber Astrid (Hg.), Acta Conventus Neo-Latini Vindobonensis / Proceedings of the Sixteenth International Congress of Neo-Latin Studies. Leiden: Brill, 2018, S. $5^{8-70 .}$

74 Genaust, Etymologisches Wörterbuch (wie Anm. 4), S. 5. 
Die Tatsache, dass die Boa in Der kleine Prinz einen Elefanten frisst und keinen Panther würgt, einmal beiseitegelassen - Genaust schildert die Suche nach der „ehedem wohl vorhandene[n] Sinnhaftigkeit der Namensgebung ${ }^{475}$ als Herausforderung, weil der Artname sowohl der Morphologie der Pflanze als auch der Kenntnis ihres natürlichen Habitats widersprechen. Dieser Widerspruch wird nicht dem entsprechenden Nomenklator zulasten gelegt (über dessen Motive auch nichts bekannt ist), sondern auf Seiten des Interpreten, sprich: des Namenslesers verortet.

Es zeigt sich also, dass die Etymologie neben ihrem unbestrittenen Nutzen als Methode (mit all ihren Beschränkungen) auch als Untersuchungsgegenstand aufschlussreich ist, zumal sie bereits in den botanischen Namensgebungsprozessen selbst eine bedeutende Rolle spielt. So nutzt Linné die Etymologie, um Bedeutung sowohl aus Pflanzennamen herauszulesen als auch in sie hineinzuschreiben, wohingegen botanische Taxa in der Forschung meist als Elemente eines reinen Verweissystems ohne jegliche semantische Ebene gelten. Einer literaturwissenschaftlich interessierten Pflanzenforschung kann es also nicht darum gehen, den Pflanzennamen einen eindeutigen Ursprung in einer bestimmten Sprache und somit eine stabile Bedeutung zuzuweisen. Vielmehr muss Etymologie im Sinne Stefan Willers als „konstruktive[r] Akt der Sprache" ${ }^{\text {"76 }}$ verstanden werden, damit botanische Texte hinsichtlich ihres poetischen Potentials untersucht werden kömmen.

Die poetische Schicht der Pflanzennamen bei Carl von Linné und anderen freizulegen, diese zu systematisieren und die Befunde auf andere Bereiche zwischen Literatur und Botanik auszuweiten (wie beispielsweise den Status einer eigenen Sprache der Botanik oder die Rolle der Blumen / Blüten zwischen Rhetorik und Systematik) wird Aufgabe einer zukünftigen literarischen Botanik sein.

75 Ebd.

76 Stefan Willer, Poetik der Etymologie: Texturen sprachlichen Wissens in der Romantik. Berlin: Akademie Verlag, 2003, S. 1 f. 